\title{
La Preescripción De Enfermería En México: Una Nueva Era
}

\author{
Nursing prescription in Mexico: A new age
}

José Arias-Rico ${ }^{a}$, Valeria Cornejo-Jovel ${ }^{b}$, Eleazar Chávez-Serrano ${ }^{c}$, Diana G. EscamillaGuillen $^{d}$, Karla I. López-Barrera ${ }^{e}$, Reyna C. Jiménez-Sánchez ${ }^{f}$, Olga R.Flores-Chávez ${ }^{g}$, Rosa M. Baltazar-Tellez ${ }^{h}$ y Barrera-Galvez Rosario ${ }^{i}$

\begin{abstract}
:
The objective of this work was to evaluate the recipe criteria. As a methodology, 10 recipes from health professionals were collected, obtained from the relatives of each one of the team members and were given statistical treatment of measures of central tendency, mean, percentage and standard deviation. As results of the analysis, we obtained that only $80 \%$ include their full name, $70 \%$ their address and telephone number, $80 \%$ professional license number, $80 \%$ the name of the institution that issued the degree, $30 \%$ refer to their specialty, $100 \%$ comply with their signature, the date the medications are prescribed and the name of the patient, $80 \%$ the patient's age, $50 \%$ the commercial name and the laboratory that produces the medication, the dosage of the medication, frequency and / or the schedule of administration of the medication, $20 \%$ the generic name and other specifications such as the circumstances in which the drug should be administered, $30 \%$ the presentation of the drug, $70 \%$ include the route of administration and the duration of the treatment, while the instructions to fill or not to recount the recipe was not found in any of the recipes analyzed, therefore it was $0 \%$. The analysis of these data is a platform for nursing students to suggest a proposal for a nursing prescription, in response to the changes to the General Health Law 2019. Concluding that not really all the prescriptions provided by health professionals health meet the requirements indicated, some of them are of utmost importance such as the personal data of the prescriber, his address and his cell phone number. An example of the nursing recipe for this new era is proposed.
\end{abstract}

Keywords:

Prescription, nursing, prescription, care

\section{Resumen:}

El objetivo de este trabajo fue evaluar los criterios de la receta. Como metodología se reunieron 10 recetas de profesionistas de la salud, obtenidas de los familiares de cada uno de los integrantes del equipo se les dio el tratamiento estadístico de medidas de tendencia central, de media, porcentaje y desviación estándar. Como resultados del análisis obtuvimos que solo el $80 \%$ incluyen su nombre completo, el $70 \%$ su domicilio y teléfono, $80 \%$ número de cédula profesional, $80 \%$ el nombre de la institución que expidió el título, $30 \%$ hacen referencia a su especialidad, el $100 \%$ cumplen con su firma, fecha en que se prescriben los medicamentos y nombre del paciente , $80 \%$ edad del paciente, $50 \%$ nombre comercial y laboratorio que produce el medicamento, dosis del medicamento frecuencia y/u horario de administración de este, el $20 \%$ el nombre genérico y otras especificaciones como las circunstancias en las que se debe de administrar el medicamento, el 30\% la presentación del fármaco, el 70\% incluye la vía de administración y el tiempo de duración del tratamiento, mientras que las instrucciones para surtir o no de nueva cuenta la receta no se encontró en ninguna de las recetas analizadas por lo tanto fue el $0 \%$. El análisis de estos datos son una plataforma para que los alumnos de enfermería puedan sugerir una propuesta de receta de prescripción de enfermería, como respuesta a los cambios a la Ley General de Salud 2019. Concluyendo que realmente que no todas las recetas brindadas por profesionales de salud cumplen los requisitos señalados, algunos de ellos son de suma importancia tal como los datos personales de quien prescribe, su domicilio y su número de celular. Se propone un ejemplo de la receta de enfermería para esta nueva era.

\section{Palabras Clave:}

Prescripción, enfermería, receta, cuidados

\footnotetext{
a. Autor de Correspondencia, Universidad Autónoma del Estado de Hidalgo, Instituto de Ciencias de la Salud, https://orcid.org/0000-0003-0219-0410, Email: josearias.rico@ hotmail.com

b. Universidad Autónoma del Estado de Hidalgo, Instituto de Ciencias de la Salud, https://orcid.org/0000-0002-4666-8490, Email: valeana2921 @ gmail.com

c. Universidad Autónoma del Estado de Hidalgo, Instituto de Ciencias de la Salud, https://orcid.org/0000-0003-2461-5037, Email: chavezeleazar465 @ gmail.com

${ }^{d}$ Universidad Autónoma del Estado de Hidalgo, Instituto de Ciencias de la Salud, https://orcid.org/0000-0002-7013-9732, Email: gerideth@ gmail.com

e Universidad Autónoma del Estado de Hidalgo, Instituto de Ciencias de la Salud, https://orcid.org/0000-0003-2574-6792, Email: ojithozmonos@ gmail.com

f. Universidad Autónoma del Estado de Hidalgo, Instituto de Ciencias de la Salud, https://orcid.org/0000-0001-9264-8514, Email: cristiji@ hotmail.com

g. Universidad Autónoma del Estado de Hidalgo, Instituto de Ciencias de la Salud, https://orcid.org/0000-0001-9479-9834, Email: ofloresc@uaeh.edu.mx

h. Universidad Autónoma del Estado de Hidalgo, Instituto de Ciencias de la Salud, https://orcid.org/0000-0002-2168-7564, Email: rbaltazar@yahoo.com

i. Universidad Autónoma del Estado de Hidalgo, Instituto de Ciencias de la Salud, ORCID: https://orcid.org/0000-0002-1949-5424, Email: rosario_barrera@uaeh.edu.mx
} 


\section{INTRODUCCIÓN}

El pasado 29 de Noviembre de 2019, se publicó la actualización de la reforma a la Ley General de Salud en el que se emiten los lineamientos que contienen el procedimiento y los criterios a los que deberán sujetarse los Licenciados en Enfermería y Pasantes de la Licenciatura en Enfermería, para la prescripción de los medicamentos. Entonces, por tratarse de intervenciones de Enfermería que pueden incurrir en implicaciones de responsabilidad legal, conviene identificar claramente las disposiciones generales de este acuerdo y la responsabilidad de los profesionales de Enfermería en el caso de imputaciones de carácter legal.

Según Pérez E. G. $(\mathbf{1 , 2})$ actualmente la facultad de prescribir medicamentos por los Licenciados en Enfermería, se encuentra regulada en el artículo 28 Bis del Reglamento de Insumos para la Salud de la Ley General de Salud, que entró en vigor el 14 de febrero de 2013. Esto implica que en la medida en que cada profesional de enfermería cumpla su labor, acorde al nivel de atención donde se desempeñe y formación académica que sustente, la calidad en los servicios de salud se verá favorecida y el usuario obtendrá mayores beneficios. Sin embargo, cualquier iniciativa al respecto deberá tener como finalidad precisar los atributos y responsabilidades que debe cumplir el personal de enfermería, en apego a las disposiciones jurídicas, normativas y fundamentos de farmacología clínica requeridos para ejecutar una prescripción de medicamentos segura.

Empezaremos por definir qué es la prescripción de Enfermería; de acuerdo a la Real Academia Española (RAE), mismo concepto que es retomado por la Dirección General de Enfermería (DGE) en el "Marco referencial para la prescripción enfermera de España".

Prescripción: Se refiere al acto del profesional de la salud que consiste en documentar a través de una receta el nombre de un fármaco, con indicaciones, dosis, vía y frecuencia en la que han de administrarse.

Receta de prescripción: Es un documento regulado a través del cual los profesionales de la salud facultados para hacerlo prescriben la medicación al paciente para que pueda adquirirla en la farmacia. Constituye un resumen del diagnóstico, pronóstico y tratamiento del padecimiento del paciente. $(\mathbf{1}, \mathbf{3})$

En esta misma línea, la receta es aquel documento normalizado por medio del cual algunos profesionales de la salud, legalmente capacitados prescriben la medicación al paciente para su dispensación por parte de las farmacias y ésta sea surtida.
Elementos básicos de una receta de prescripción:

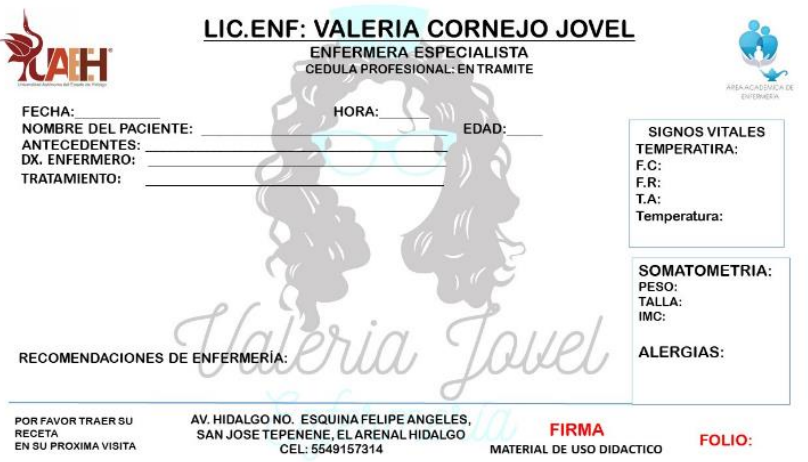

Figura 1. Ejemplo de receta de enefermería como resultado de la práctica.

La receta de prescripción es una orden escrita emitida por el profesional de la salud para que se surta o dispense uno o varios medicamentos al paciente. La elaboración de una receta sigue un esquema definido, por lo que la ley en salud señala los requisitos que ésta debe contener, los cuales se indican a continuación:

Datos obligatorios impresos:

* Nombre de quien prescribe

* Domicilio completo de quien prescribe Número de cédula profesional del médico ( $\mathrm{y}$ de especialidad en su caso)

- Nombre de la Institución que otorgo el título profesional de medicina general (y de especialidad en su caso)

- Especialidad del Médico o Enfermero

Información Obligatoria (manuscrita o de computadora):

* Fecha de emisión de la receta.

* Firma autógrafa del médico (manuscrita).

* Nombre y edad del paciente.

* Denominación genérica y en su caso, distintiva del medicamento.

* Dosis.

* Presentación comercial (indicar forma farmacéutica, contenido y concentración del medicamento).

* Vía de administración. Frecuencia.

* Tiempo de duración del tratamiento.

* Cantidad por surtir (no más de 2 piezas del mismo medicamento para fracción Il y la indicada por el médico en caso de fracción III)

- Para recetas de instituciones públicas o privadas, sello de la institución.

Para los medicamentos clasificados en las fracciones II y III, se debe elaborar una receta ordinaria por separado para cada medicamento. No deberán prescribirse 
medicamentos controlados de los distintos grupos (I, II y III) en una sola receta, la receta de medicamentos de la fracción II será exclusiva para la entrega de hasta dos presentaciones (piezas) del mismo medicamento, la receta de medicamentos correspondientes a la fracción III podrá surtirse hasta en tres ocasiones dentro de los 6 meses siguientes a la fecha de emisión.

El dato de Diagnóstico, es OBLIGATORIO en las recetas especiales del Grupo I de medicamentos (Estupefacientes), pero NO ES INDISPENSABLE para las recetas médicas de los Grupos II y III (Psicotrópicos) así como de antibióticos; por lo que tal dato puede ser omitido en las recetas que usted reciba (de los grupos II, III y IV) de tal modo que las farmacias podrán surtir la receta sin inconvenientes. $(4,5)$

Lo anterior son los elementos necesarios para poder elaborar una receta médica, en el caso de enfermería son los mismos requisitos, pero claramente no se pueden recetar los mismos medicamentos que los de un médico. La receta médica necesita tener todos los aspectos anteriores para poder recetar correctamente a un paciente.

No olvidemos que la receta se debe tomar como algo serio y de beneficio para el área de enfermería, ya que es un nivel más ante la sociedad. Lo que queda es enseñar a los alumnos del área de enfermería a prescribir y formular correctamente una receta médica, para que en su vida profesional le sea de utilidad.

\section{Aspectos legales a tomarse en cuenta}

Según la Ley de Profesiones, $(\mathbf{1 , 6 )}$ los Licenciados en Enfermería deben contar con cédula para su ejercicio profesional, según se señala en el artículo 28 - Bis de la Ley General de Salud $(1,7)$ Así, la Ley de Profesiones también señala en el Art.3. . . podrá obtener cédula de ejercicio quien legalmente haya obtenido un título profesional. En el Art. 29 dice: Las personas que sin tener título profesional legalmente expedido actúen habitualmente como profesionistas, incurrirán en las sanciones que establece esta Ley, En el Art. 30. Autorización a los pasantes de las diversas profesiones para ejercer la práctica respectiva por un término no mayor de tres años, y en el Art. 61. Los delitos que cometan los profesionistas en el ejercicio de la profesión, serán castigados por las autoridades competentes con arreglo al Código Penal. El objetivo de esta práctica fue conocer los elementos que debe llevar una receta comparar con las rectas recolectadas y así el alumno elabore un diseño para su futura receta

\section{METODOLOGÍA}

Éste trabajo es el resultado de una práctica de laboratorio realizada como parte de la asignatura de introducción a la farmacología. En equipo de 5 personas se recolectaron 10 recetas de diferentes médicos o clínicas para la revisión en clase. Se analizaron cada una para evaluar si cumplían con la Ley General de Salud. Se manejaron los datos en tabla de frecuencias y porcentajes. Los alumnos en base a lo observado diseñaron su propia receta para que en un futuro sea su receta de enfermería (figura1).

\section{RESULTADOS}

Al revisar cada una de las recetas se arroja la siguiente información. En la tabla 1 se observa que en su mayoría cumplió al $100 \%$ con los requisitos de una receta destacando que en un $50 \%$ se cumplió los rubros de ficha del paciente, nombre comercial y presentación del medicamento. El tiempo de duración del tratamiento ocurrió en un $20 \%$.

Tabla 1. Análisis de receta

\begin{tabular}{lcc}
\hline \multicolumn{1}{c}{ Especificaciones } & $\mathbf{n}$ & $\%$ \\
\hline Fechas & 10 & 100 \\
Ficha del paciente & 5 & 50 \\
Nombre & 10 & 100 \\
Edad & 8 & 80 \\
Peso & 4 & 40 \\
El símbolo de prescripción & 6 & 60 \\
Medicamentos & 10 & 100 \\
Nombre comercial y & & \\
laboratorio que produce el & 5 & 50 \\
medicamento & & \\
Nombre genérico & 2 & 20 \\
$\begin{array}{l}\text { Presentación: Forma } \\
\text { farmacéutica y cantidad del } \\
\text { principio activo }\end{array}$ & 3 & 30 \\
$\begin{array}{l}\text { Instrucciones de uso } \\
\text { Dosis }\end{array}$ & 8 & 80 \\
Vía de administración & 3 & 30 \\
$\begin{array}{l}\text { Frecuencia y/u horario de } \\
\text { administración }\end{array}$ & 7 & 70 \\
$\begin{array}{l}\text { Tiempo de duración del } \\
\text { tratamiento }\end{array}$ & 6 & 60 \\
$\begin{array}{l}\text { Otras especificaciones } \\
\text { Instrucciones para surtir o no }\end{array}$ & 7 & 70 \\
\hline
\end{tabular}

Fuente: Recetas de prescripción llevadas por los alumnos 2020.

También se realizó una gráfica en la que se observa que solo el $30 \%$ de las recetas cumplieron con más del $75 \%$ de las especificaciones de una receta (gráfica 1) 


\section{Gráfica 1. Recetas de prescripción}

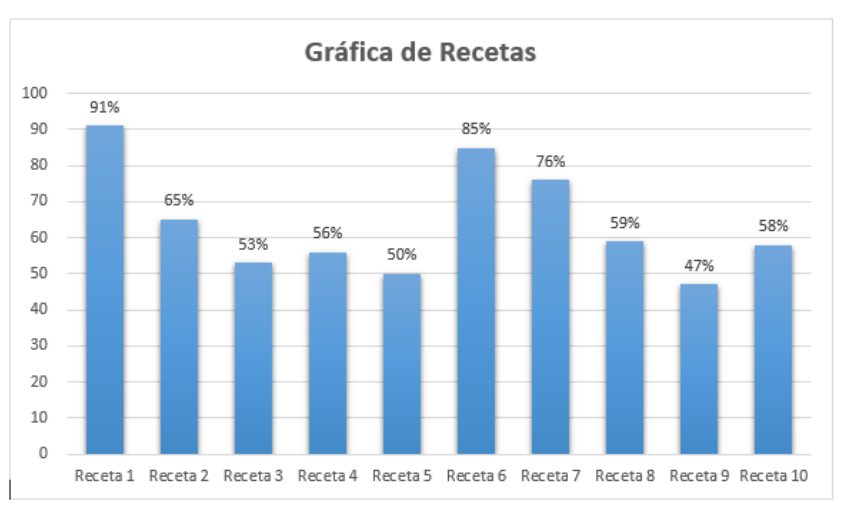

Se deduce que las recetas 1 y 6 son las mejores de toda la encuesta, estas son tomadas de una institución de salud pública y profesionistas de la salud particulares. Estas además de cumplir con varios puntos a favor se puede apreciar que las recetas institucionales son de mayor precisión que las de la consulta privada como es el caso de las recetas 9 y 5 que son las más bajas. La importancia de que una receta cumpla con todos los puntos anteriores es vital para un buen tratamiento y la mejor recuperación del paciente. Además de que es la forma de presentación de un médico o enfermero y también habla de su calidad de trabajo.

\section{CONCLUSIÓN}

Ser enfermero hoy en día es una responsabilidad muy grande porque aparte de cuidar y ver por el bien del paciente también hoy en día se cuida la integridad y el bienestar desde el punto de vista farmacológico.

En México el ser responsable de una vida conlleva desde grandes esperanzas y agradecimientos hasta demandas. Es por esto que el área de enfermería debe de estar lo más capacitada posible y así poder ayudar en vez de afectar. La elaboración de una receta necesita de un conocimiento previo para poder ser funcional y además el personal también necesita de una capacitación previa para poder saber que se puede recetar y que no.

Las generaciones nuevas de enfermería necesitan llevar este aprendizaje, para poder usar la nueva herramienta y mejorar las condiciones de los pacientes.

Por ultimo hay que reconocer que se espera mucho de esta nueva herramienta en el área de enfermería y lo más importante es el saber utilizarlo de la mejor manera y con responsabilidad.

\section{REFERENCIAS}

1. Lasty, B. La prescripción de los medicamentos por los licenciados en enfermería: implicaciones de responsabilidad legal. 2017, de REVISTA CONAMED Sitio web: file://C:/Users/User/Downloads/DialnetLaPrescripcionDeLosMedicamentosPorLosLicenciadosEn -6434803.pdf
2. Pérez E. G. Prescripción por profesionales de Enfermería en México. Editorial. Enfermería del IMSS. México, 2013; 21 (2): $61-62$.

3. Consejo General de Enfermería. Marco referencial para la prescripción enfermera. España 2008. Disponible en: http://www.index-f.com/lascasas/documentos/lc0151.pdf

4. Emmanuel O, Cerezo E, Amador AT. Elaboración y diseño de la receta de Enfermería. 2016., 5.

5. Aguascalientes G. Requisitos Receta Medica. Gob Aguascalientes [Internet]. 2018;1:242. Available from: https://www.issea.gob.mx/Docs/Regulacion Sanitaria/Requisitos Receta Médica.pdf

6. Cámara de Diputados. Ley de Profesiones. México, 2017. Disponible en: http://www.diputados.gob.mx/LeyesBiblio/ pdf/208.pdf Consultado el día 31 de Mayo del 2017.

7. Maschinen $B$, Investition A, Beschaffungen G, Ersatzbeschaffungen $B$, Mittelherkunft $S$. Planes de Cuidados Enfermeros en SM [Internet]. Salud C de $S$ y $S$ de, editor. Extremadura, España; 2008. 3-90 p. Available from:

https://saludextremadura.ses.es/filescms/smex/uploaded_f iles/CustomContentResources/Planes_de_Cuidados_Enfe rmeros_en_Salud_Mental.pdf

8. Consejo General de Enfermería. Marco referencial para la prescripción enfermera. Consj Gral Enf [Internet]. 2006;2543. Available from: http://www.indexf.com/lascasas/documentos/lc0151.pdf

9. Beca Infante J, Ortiz Pommier A. Etica De La Receta Medica. 2008;4. Available from: https://medicina.udd.cl/centrobioetica/files/2010/10/receta-médica.pdf 